\title{
Rarefied Aerothermodynamic Predictions for Mars Global Surveyor
}

\author{
R. G. Wilmoth ${ }^{1}$, D. F. G. Rault ${ }^{1}$, R. W. Shane ${ }^{2}$, R. H. Tolson ${ }^{2}$ \\ ${ }^{1}$ NASA Langley Research Center, Hampton, VA, USA \\ ${ }^{2}$ George Washington University, Hampton, VA, USA
}

\section{Introduction}

Mars Global Surveyor (MGS) successfully completed its first phase of aerobraking in early 1998 and is the first planetary mission to use aerobraking as a primary means of customizing its orbit to achieve its mission objectives [1]. The aerobraking requirements together with post-launch anomalies [2] presented a unique challenge to provide accurate predictions of the aerothermodynamic environment of the spacecraft in the rarefied transitional flow regime. Direct Simulation Monte Carlo (DSMC) and freemolecular techniques were used to provide heating and aerodynamic predictions and to investigate a variety of rarefied flow phenomena across the flight regime $[3,4,5]$; MGS is the first major planetary mission in which rarefiedflow predictions have played such a critical role all the way through the design, mission planning, and operational phases. This paper summarizes these studies with emphasis on transitional-flow and gas-surface interaction phenomena.

\section{Computational Approach}

Analyses were carried out using the NASA LaRC 3D DSMC (LaRC-3D) code [6], the DAC (DSMC Analysis Code) code [7], and companion 3D freemolecular codes developed in recent years for analyzing complex geometries. The DSMC codes differ primarily in their computational grid methodology and modeling of the surface geometry. However, both DSMC codes use essentially the same DSMC procedures and physical models and are capable of handling the complex geometry of the MGS spacecraft. The DSMC codes are complemented by $3 \mathrm{D}$ free-molecular codes which use analytical freemolecular analysis and line-of-sight shadowing techniques to model the flow about complex geometries. Analysis of MGS aerodynamics and heating was performed primarily using LaRC-3D in the design phase and in the early 
post-launch, pre-aerobraking phase, while DAC was used primarily in the later post-launch, pre-aerobraking phase and in the early operational phase of aerobraking.

Calculations were performed for a variety of solar panel positions and spacecraft attitudes and for atmospheric densities ranging from free-molecular to transitional flow (Knudsen numbers $<0.1$ ). The composition of the Mars atmosphere was assumed to be $95.37 \% \mathrm{CO}_{2}$ and $4.63 \% \mathrm{~N}_{2}$ by mole. Collisions were modeled with the Variable Hard Sphere model of Bird[8] using a reference temperature of $3000 \mathrm{~K}$, a temperature exponent of 0.806638 , and reference diameters of $3.8969 \times 10^{-10} \mathrm{~m}$ and $3.3487 \times 10^{-10} \mathrm{~m}$ for $\mathrm{CO}_{2}$ and $\mathrm{N}_{2}$, respectively. Gas-surface interactions were modeled as a combination of diffuse and specular scattering. The effects of $\mathrm{CO}_{2}$ evaporation were studied and modeled as a simple steady-state outgassing process.

\section{$3 \quad$ Spacecraft Geometry and Modeling Issues}

The geometry of the MGS spacecraft in the aerobraking configuration is shown in Fig. 1. The solar panels are swept back to reduce aerodynamic heating and to provide aerodynamic stability. The pre-launch design called for both panels to be swept 30 deg to give essentially a symmetric configuration. However, when the panels were deployed after launch, the $-\mathrm{Y}$ panel failed to fully extend due to what was determined to be a jammed hinge between the yoke and the inner solar panel [2]. This failure left the -Y panel approximately 20 deg short of full deployment and resulted in a configuration that would not have been sufficiently stiff when subjected to aerodynamic moments about the $\mathrm{X}$-axis. The problem was resolved by rotating the -Y panel $180 \mathrm{deg}$ about its principal axis and sweeping the yoke $51 \mathrm{deg}$ aft to compensate for the $20 \mathrm{deg}$ shortfall in panel deployment. The $+\mathrm{Y}$ panel of this revised aerobraking configuration was swept to approximately 33.8 deg to provide the desired aerodynamic trim about the $\mathrm{X}$-axis. The revised configuration also provided greater stiffness to aerodynamic moments about the $\mathrm{X}$-axis although small deflections of the $-\mathrm{Y}$ panel about the unlatched hinge were expected. These deflections were computed and taken into account when extracting atmospheric density from the on-board accelerometer measurements [9].

\section{Results}

\subsection{Aerodynamic Heating}

One of the early design considerations was the aerodynamic heating that would occur on the solar panels during aerobraking. Although the estimated 
Marseille, France, July 26-31, 1998

heating levels were relatively low, the allowable temperatures on the solar panel still limited the depth to which the spacecraft could penetrate the Martian atmosphere without exceeding these temperatures. The maximum atmospheric densities expected at periapsis placed the spacecraft well into transitional flow, and DSMC analyses were conducted to refine the predicted heating levels.

Figure 2 shows density contours around MGS at densities of 12 and 120 $\mathrm{kg} / \mathrm{km}^{3}$ where the higher value is approximately the maximum expected density at periapsis. These predictions exhibit the typical diffuse shock layers that occur in rarefied, transitional flows. The shock layer effectively "shields" the body from receiving the full kinetic energy of freestream molecules, and local heating rates are significantly lower than free-molecular values as seen in Fig. 3. This figure shows heat transfer coefficients, $C_{H}$ $\left(\equiv \dot{q} /\left(\frac{1}{2} \rho_{\infty} V_{\infty}^{3}\right)\right)$, along a diagonal cut on the windward side of the inboard solar panel, and reductions up to $40 \%$ are seen near the center of the panel at the highest density. Such predictions were important for "calibrating" temperature sensors on the panels that were used to assess the actual heating levels in flight.

\subsection{Drag During Aerobraking}

Aerobraking depends on aerodynamic drag to slow the spacecraft by a small velocity increment, $\Delta V$, on each pass. The total reduction in velocity required is determined by the final orbit one wishes to achieve. The $\Delta V$ obtained on each aerobraking pass is directly proportional to the atmospheric density and the drag coefficient of the spacecraft. To minimize the time required to achieve final orbit, it is desirable to penetrate as deeply as possible into the atmosphere and to maximize the drag coefficient. Figure 4 shows predictions of the drag coefficient over the density range of interest for MGS. Predictions for the symmetric sweep pre-launch configuration obtained with LaRC-3D and the revised sweep configuration obtained with DAC are shown to demonstrate that drag variations for the two configurations are essentially the same. The drag coefficient is reduced up to $15 \%$ at the highest density, and although this reduction may seem small, its effect on the total number aerobraking passes required can be significant. Therefore, DSMC predictions were important in the early design to provide accurate predictions of the drag coefficient of MGS.

The impact of drag on aerobraking time is seen in Fig. 5 which shows a history of MGS orbit parameters at periapsis over approximately the first eight months of operation. The original mission called for a target orbit period of approximately two hours to be achieved in about 120 days. (The exact time required was subject to relatively large uncertainties in atmospheric density.) However, excessive deflections of the partially-deployed -Y 
solar panel during the first few weeks caused a 30-day halt to aerobraking while these deflections were analyzed. When aerobraking resumed, it was decided to minimize the panel loads by aerobraking at lower densities and to extend the total aerobraking phase to about 18 months which includes a six-month non-aerobraking period for obtaining science data.

\subsection{Aerodynamic Solar Panel Deflections}

The partially-deployed -Y panel was not "latched" into a rigid position, and deflections were expected during aerodynamic loading. These deflections were also expected to reduce the drag coefficient and alter the aerodynamic trim. Therefore, additional analyses were performed to provide a more complete aerodynamic database for various panel deflections, and two of the more important quantities, the drag coefficient, $C_{D}$, and the yawing moment about the X-axis, $C_{m, x}$, are shown in Fig. 6 for panel deflections of up to $20 \mathrm{deg}$. Drag reductions of $10-15 \%$ are predicted at the maximum panel deflection, and trim angle, $\beta$, about the $\mathrm{X}$-axis is shifted by about one-half of the deflection angle. These predictions were used to extract atmospheric densities from the onboard accelerometer data during each aerobraking pass, and an iterative procedure was required to compute the panel deflection consistent with the axial force coefficient, $C_{z}$, corresponding to that deflection. Figure 7 shows typical axial force coefficients and computed panel deflections derived from flight data, and the deflections show relatively good agreement with independent measurements of deflections made with an onboard sun sensor [9].

\subsection{Gas-Surface Interaction Effects}

\subsubsection{Momentum Accommodation}

The revised aerobraking configuration placed the glass-covered solar cells of the -Y panel on the windward side, and differences in momentum accommodation coefficients between the glass and the composite material on the windward side of the $+\mathrm{Y}$ panel were expected. The main effect of these accommodation coefficient differences was expected to be a change in aerodynamic trim about the $\mathrm{X}$-axis. Therefore, free-molecular calculations were first performed for accommodation coefficient differences of up to 0.3 (actual differences were expected to be less than 0.2), and the predicted shift in yaw trim angle is shown in Fig. 8. Although the predicted shifts are small, they were important in assessing panel deflections in the early passes, and a few DSMC calculations were made at a nominal density of $81 \mathrm{~kg} / \mathrm{km}^{3}$ to further refine the predictions. At this transitional flow density, the effect of accommodation differences is diminished considerably because of molecular collisions near the surface that cause partially accommodated molecules to 
have multiple surface collisions.

\subsection{2 $\mathrm{CO}_{2}$ Evaporation}

Accelerometer flight measurements have shown perturbations in axial acceleration during certain aerobraking passes that cannot be explained by known atmospheric density perturbations or by spacecraft geometry. One explanation considered is that the "cold" solar panels might adsorb sufficient $\mathrm{CO}_{2}$ which then rapidly evaporates during aerodynamic heating, and this effect has been qualitatively evaluated using a simple "outgassing" model. Evaporation of $\mathrm{CO}_{2}$ was modeled on the windward surfaces of the solar panels at various arbitrary rates to determine the effect on axial force, and the results are shown in Fig. 9. Evaporation flux rates of up to four times the incident freestream flux were simulated at the relatively rarefied freestream density of $12 \mathrm{~kg} / \mathrm{km}^{3}$. Figure 9 shows the net change in axial force and the components caused by the "shielding" effect produced by evaporating molecules colliding with freestream molecules (a reduction in axial force or drag) and the "thrust" exerted by the evaporating molecules as they leave the surface. These effects can be qualitatively understood by examining the streamlines around the spacecraft shown in Fig. 10 which clearly show the "shielding" effect as well as the streamlines emanating from the surface.

The evaporation rates simulated in this brief study did not produce sufficient changes in axial force to explain the observed flight data. Furthermore, estimates of the quantity of $\mathrm{CO}_{2}$ that might be adsorbed suggest that evaporation rates higher than those simulated are unlikely, although the model used to make these estimates is subject to large uncertainties due to uncertainties in surface phase effects and heat of evaporation.

\section{Concluding Remarks}

Mars Global Surveyor has been highly successful in demonstrating that aerobraking can be used to tailor a planetary orbit, and rarefied flow simulations have contributed significantly to the successful design, mission planning, and flight operations. Engineering flight data obtained to date has provided added confidence in the aerothermodynamic predictions, and both the engineering and science data are providing a wealth of information on planetary aerobraking and on the Mars environment.

\section{References}

[1] Dallas, S. Sam, Mars Global Surveyor Mission, Proceedings of IEEE Aerospace Conference, Vol. 4, Snowmass at Aspen, CO, Feb. 1-8, 1997, pp. $173-189$. 
[2] Lyons, Daniel T., Mars Global Surveyor: Aerobraking with a Broken Wing, AAS Paper 97-618, Aug. 1997.

[3] Rault, D. F. G., RCS Plume Effect on Spacecraft Aerodynamics, 20th Rarefied Gas Dynamics Symposium, Bejiing, China, August, 1996.

[4] Shane, R. W, Rault, D. F. G., and Tolson, R. H., Mars Global Surveyor Aerodynamics for Maneuvers in Martian Atmosphere, AIAA Paper 972509, June, 1997.

[5] Wilmoth, R. G., Rault, D. F. G., Cheatwood, F. M., Engelund, W. C., and Shane, R. W., Rarefied Aerothermodynamics Predictions of Mars Global Surveyor, Submitted to Journal of Spacecraft and Rockets, 1998.

[6] Rault, D. F. G., Towards an Efficient Three-Dimensional DSMC code for Complex Geometry Problems, 18th Rarefied Gas Dynamics Symposium, Vancouver, British Columbia, July 1992.

[7] Wilmoth, R. G., LeBeau, G. J.; and Carlson, A. B., DSMC Grid Methodologies for Computing Low-Density, Hypersonic Flows About Reusable Launch Vehicles, AIAA Paper 96-1812, June 1996.

[8] Bird, G. A., Molecular Gas Dynamics and the Direct Simulation of Gas Flows, Clarendon Press, Oxford, 1994.

[9] Tolson, R. H., Keating, G. M., Cancro, G. J., Parker, J. S., Noll, S. N., and Wilkerson, B. L, Application of Accelerometer Data to Mars Global Surveyor Aerobraking Operations, Submitted to Journal of Spacecraft and Rockets, 1998.

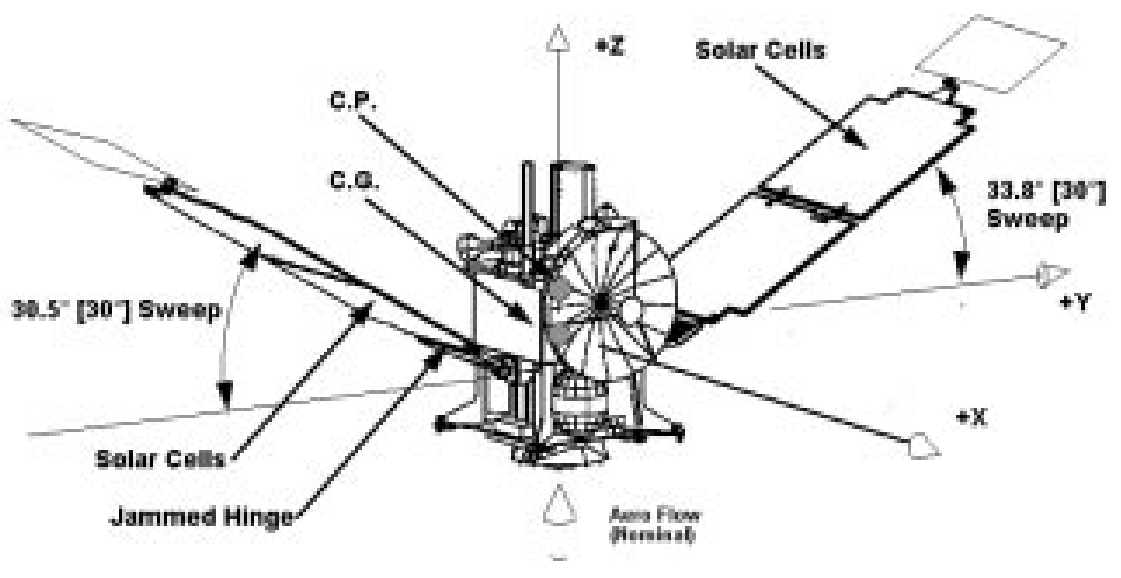

Figure 1: MGS Spacecraft in Aerobraking Configuration. 


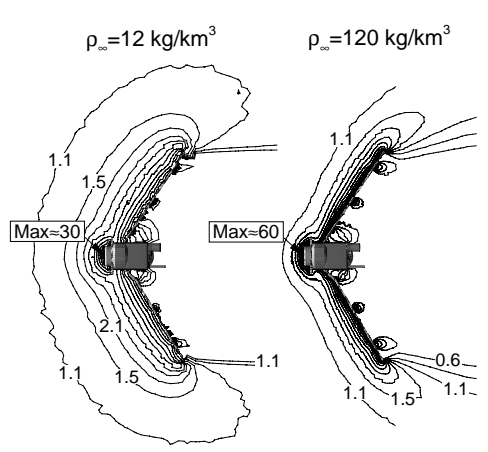

Figure 2: Nondimensionalized Density Contours, $\rho / \rho_{\infty}$.

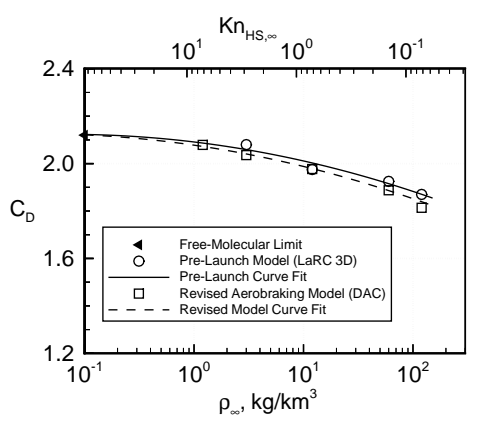

Figure 4: Drag Predictions for Various Geometry Models and DSMC Codes.

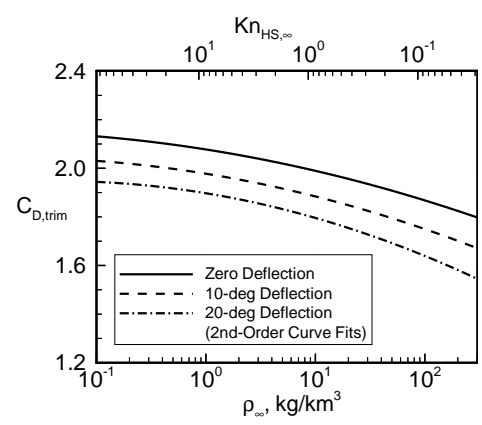

(a) Drag Coefficient.

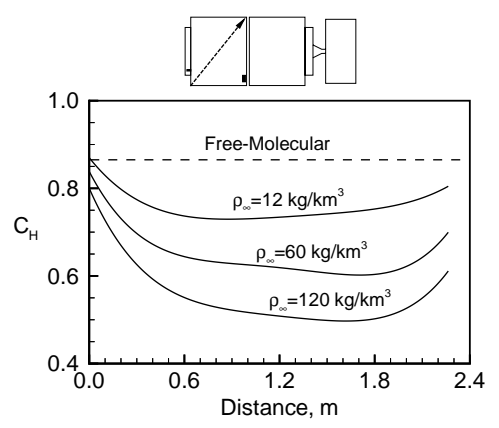

Figure 3: Transitional Effect on Solar Panel Heat Transfer.

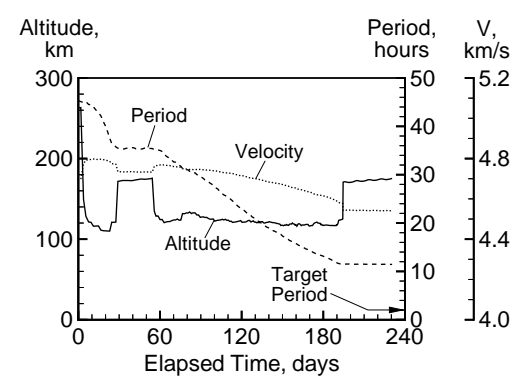

Figure 5: MGS Aerobraking History. (Values given at periapsis.)

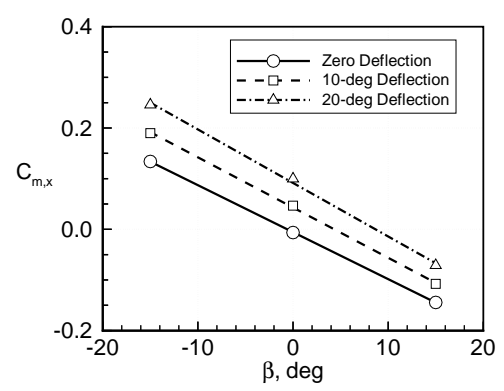

(b) Yawing Moment.

Figure 6: Effect of -Y Panel Deflection. 


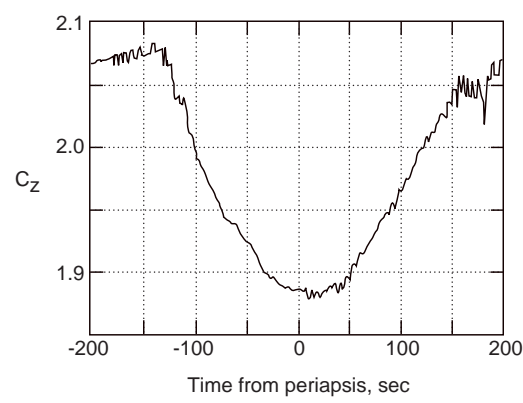

(a) Axial Force Coefficient.

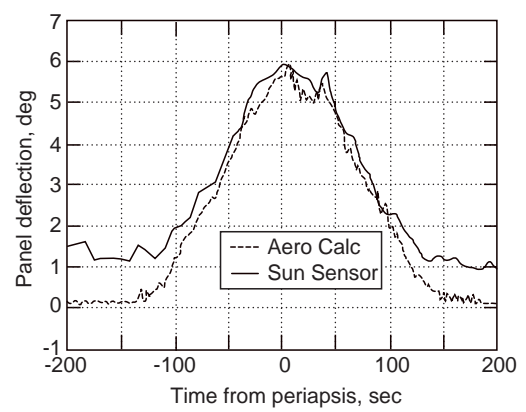

(b) Measured and Calculated Panel Deflection.

Figure 7: Axial Force and Deflections Extracted from Flight Data.

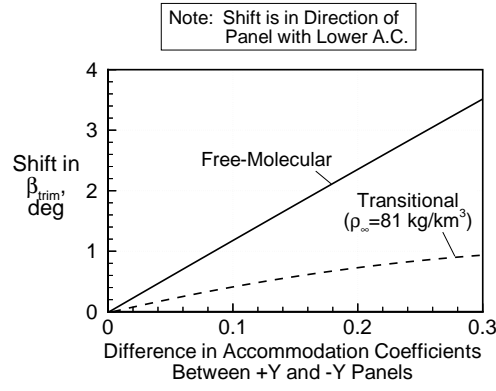

Figure 8: Effect of Differential Accommodation on Yaw Trim.

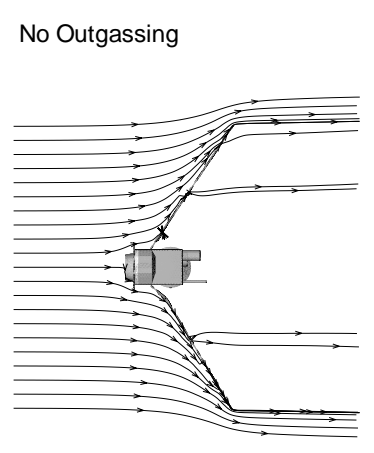

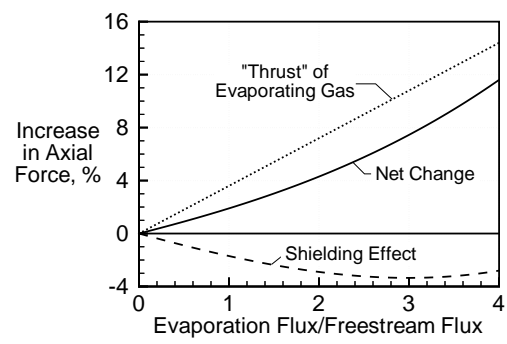

Figure 9: Effect of $\mathrm{CO}_{2}$ Evaporation on Axial Force.

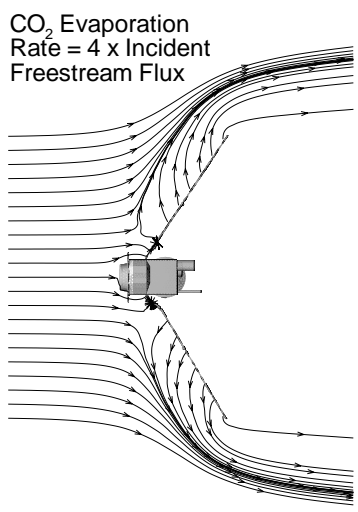

Figure 10: Effect of $\mathrm{CO}_{2}$ Evaporation on Streamlines. $\rho_{\infty}=12 \mathrm{~kg} / \mathrm{km}^{3}$. 